\title{
ON THE COMPUTATION OF BATTLE-LEMARIÉ'S WAVELETS
}

\author{
MING-JUN LAI
}

\begin{abstract}
We propose a matrix approach to the computation of BattleLemarie's wavelets. The Fourier transform of the scaling function is the product of the inverse $F(\mathbf{x})$ of a square root of a positive trigonometric polynomial and the Fourier transform of a B-spline of order $m$. The polynomial is the symbol of a bi-infinite matrix $B$ associated with a B-spline of order $2 m$. We approximate this bi-infinite matrix $\mathbf{B}_{2 m}$ by its finite section $A_{N}$, a square matrix of finite order. We use $A_{N}$ to compute an approximation $\mathbf{x}_{N}$ of $\mathbf{x}$ whose discrete Fourier transform is $F(\mathbf{x})$. We show that $\mathbf{x}_{N}$ converges pointwise to $\mathbf{x}$ exponentially fast. This gives a feasible method to compute the scaling function for any given tolerance. Similarly, this method can be used to compute the wavelets.
\end{abstract}

\section{INTRODUCTION}

Battle-Lemarie's wavelets $[1,3]$ may be constructed by using a multiresolution approximation built from polynomial splines of order $m>0$. See, e.g., [4] or [2]. To be precise, let $V_{0}$ be the vector space of all functions of $L^{2}(\mathbf{R})$ which are $m-2$ times continuously differentiable and equal to a polynomial of degree $m-1$ on each interval $[n+m / 2, n+1+m / 2]$ for all $n \in \mathbf{Z}$. Define the other resolution space $V_{k}$ by

$$
V_{k}:=\left\{u\left(2^{k} t\right): u \in V_{0}\right\}, \quad \forall k \in \mathbf{Z} .
$$

It is known that $\left\{V_{k}\right\}_{k \in \mathbf{Z}}$ provide a multiresolution approximation, and there exists a unique scaling function $\varphi$ such that

$$
V_{k}=\operatorname{span}_{L^{2}}\left\{2^{k / 2} \varphi\left(2^{k} t-n\right): n \in \mathbf{Z}\right\}
$$

for all $k$, and the integer translates of $\varphi$ are orthonormal to each other. (See, e.g., [4].) Define a transfer function $H(\omega)$ by

$$
H(\omega)=\frac{\hat{\varphi}(2 \omega)}{\hat{\varphi}(\omega)},
$$

where $\hat{\varphi}$ denotes the Fourier transform of $\varphi$. Then the wavelet $\psi$ associated with the scaling function $\varphi$ is given in terms of its Fourier transform by

$$
\hat{\psi}(\omega)=e^{-j \omega / 2} \bar{H}(\omega / 2+\pi) \hat{\varphi}(\omega / 2) .
$$

Received by the editor May 14, 1993.

1991 Mathematics Subject Classification. Primary 41A15, 41A30, 42C15, 47B35.

Key words and phrases. B-spline, bi-infinite matrices, exponential decay, finite section, positive operator, Toeplitz matrix, wavelet.

Research supported by the National Science Foundation under Grant \#DMS-9303121. 
Here and throughout, $j:=\sqrt{-1}$. The scaling function $\varphi$ associated with the multiresolution approximation may be given by

$$
\hat{\varphi}(\omega)=\frac{1}{\sqrt{\sum_{k \in \mathbf{Z}}\left|\widehat{B}_{m}(\omega+2 k \pi)\right|^{2}}} \widehat{B}_{m}(\omega),
$$

where $B_{m}$ is the well-known central B-spline of order $m$ whose Fourier transform is given by

$$
\widehat{B}_{m}(\omega)=\left(\frac{\sin \omega / 2}{\omega / 2}\right)^{m} .
$$

By using Poisson's summation formula, we have

$$
\hat{\varphi}(\omega)=\frac{1}{\sqrt{\sum_{k \in \mathbf{Z}} B_{2 m}(k) e^{-j k \omega}}} \widehat{B}_{m}(\omega) .
$$

Thus, the transfer function is

$$
H(\omega)=\sqrt{\frac{\sum_{k \in \mathbf{Z}} B_{2 m}(k) e^{-j k 2 \omega}}{\sum_{k \in \mathbf{Z}} B_{2 m}(k) e^{-j k \omega}}}(\cos \omega / 2)^{m} .
$$

Then the wavelet $\psi$ associated with $\varphi$ is given by

$$
\hat{\psi}(\omega)=e^{-j \omega / 2} \overline{H(\omega / 2+\pi)} \frac{1}{\sqrt{\sum_{k \in \mathbf{Z}} B_{2 m}(k) e^{-j k \omega / 2}}} \widehat{B}_{m}(\omega / 2) .
$$

The above Fourier transforms of $\varphi, H$, and $\psi$ suggest that the scaling function, transfer function, and wavelet have the following representations:

$$
\begin{aligned}
\varphi(t) & =\sum_{k \in \mathbf{Z}} \alpha_{k} B_{m}(t-k), \\
H(\omega) & =\sum_{k \in \mathbf{Z}} \beta_{k} e^{-j k \omega}, \\
\psi(t) & =\sum_{k \in \mathbf{Z}} \gamma_{k} B_{m}(2 t-k) .
\end{aligned}
$$

In this paper, we propose a matrix method to compute the $\alpha_{k}$ 's, $\beta_{k}$ 's, and $\gamma_{k}$ 's. Let us use $\varphi$ to illustrate our method as follows: view $\sum_{k \in \mathbf{Z}} B_{2 m}(k) e^{-j k \omega}$ as the symbol of a bi-infinite matrix $\mathbf{B}_{2 m}=\left(b_{i k}\right)_{i, k \in \mathbf{Z}}$ with $b_{i, k}=b_{0, k-i}=$ $B_{2 m}(k-i)$ for all $i, k \in \mathbf{Z}$. Similarly, $\sqrt{\sum_{k \in \mathbf{Z}} B_{2 m}(k) e^{-j k \omega}}$ can be viewed as the symbol of another (unknown) bi-infinite matrix $C_{2 m}$. Then it is easy to see that

To find

$$
\mathbf{C}_{2 m}^{2}=\mathbf{B}_{2 m} .
$$

$$
\sum_{k \in \mathbf{Z}} \alpha_{k} e^{-j k \omega}=\frac{1}{\sqrt{\sum_{k \in \mathbf{Z}} B_{2 m}(k) e^{-j k \omega}}}
$$

is equivalent to solving

$$
C_{2 m} \mathbf{x}=\delta
$$

with $\delta=\left(\delta_{i}\right)_{i \in \mathbf{Z}}, \delta_{0}=1$, and $\delta_{i}=0$ for all $i \in \mathbf{Z} \backslash\{0\}$, where $\mathbf{x}=\left(\alpha_{k}\right)_{k \in \mathbf{Z}}$. Our numerical method is to find an approximation to $\mathbf{x}$ within a given tolerance. 
Let $A_{N}=\left(b_{i k}\right)_{-N \leq i, k \leq N}$ be a finite section of $\mathbf{B}_{2 m}$. Note that $A_{N}$ is symmetric and totally positive. Thus, we can find $\widehat{P}_{N}$ such that

$$
\widehat{P}_{N}^{2}=A_{N}
$$

by using, e.g., the singular value decomposition. Then we solve $\widehat{P}_{N} \mathbf{x}_{N}=\delta_{N}$ with $\delta_{N}$ a vector of $2 N+1$ components which are all zeros except for the middle one, which is 1 . We can show that $\mathbf{x}_{N}$ converges pointwise to $\mathbf{x}$ exponentially fast. Similarly, we can use this idea to compute an approximation of $\left\{\boldsymbol{\beta}_{k}\right\}_{k \in \mathbf{Z}}$ by (2) and $\left\{\gamma_{k}\right\}_{k \in Z}$ by (3). Therefore, the discussion mentioned above furnishes a numerical method to compute Battle-Lemarié's wavelet.

To prove the convergence of $\mathbf{x}_{N}$ to $\mathbf{x}$, we place ourselves in a more general setting. We study a general bi-infinite matrix $A$ : (For the case of BattleLemarié's wavelets, $A=\mathbf{B}_{2 m}$.) We look for certain conditions on $A$ such that the solution $\mathbf{x}_{N}$ of $\widehat{P}_{N} \mathbf{x}_{N}=\delta_{N}$ with $\widehat{P}_{N}^{2}=A_{N}$ converges to the solution $\mathbf{x}$ of $P \mathbf{x}=\delta$ with $P^{2}=A$, where $A_{N}$ is a finite section of $A$. This is discussed in the next section. In the last section, we show that the bi-infinite matrix $\mathbf{B}_{2 m}$ satisfies the conditions on $A$ obtained in $\S 2$. This will establish our numerical method for computing Battle-Lemarié's wavelets.

\section{MAIN Results}

Let $\mathbf{Z}$ be the set of all integers. Let $l^{2}:=l^{2}(\mathbf{Z})$ be the space of all square summable sequences with indices in $\mathbf{Z}$. That is,

$$
l^{2}(\mathbf{Z})=\left\{\left(\ldots, x_{-1}, x_{0}, x_{1}, \ldots\right)^{t}: \sum_{i=-\infty}^{\infty}\left|x_{i}\right|^{2}<\infty\right\} .
$$

It is known that $l^{2}$ is a Hilbert space. We shall use $\mathbf{x}$ to denote each vector in $l^{2}$ and use $A$ to denote a linear operator from $l^{2}$ to $l^{2}$. It is known that $A$ can be expressed as a bi-infinite matrix. Thus, we shall write $A=\left(a_{i k}\right)_{i, k \in \mathbf{Z}}$.

In the following, we shall consider $A$ to be a banded and/or Toeplitz matrix. That is, $A$ is said to be banded if there exists a positive integer $b$ such that $a_{i k}=0$ whenever $|i-k|>b$. The matrix $A$ is said to be Toeplitz if $a_{i+k, m+k}=$ $a_{i, m}$ for all $i, k, m \in \mathbf{Z}$. Denote by $F(\mathbf{x})(\omega)$ the symbol of a vector $\mathbf{x} \in l^{2}$, i.e.,

$$
F(\mathbf{x})(\omega)=\sum_{i \in \mathbf{Z}} x_{i} e^{-j i \omega} .
$$

Denote by $F(A)(\omega)$ the symbol of a Toeplitz matrix $A=\left(a_{i k}\right)_{i, k \in \mathbf{Z}}$, i.e.,

$$
F(A)(\omega)=\sum_{i \in \mathbf{Z}} a_{i, 0} e^{-j i \omega} .
$$

Suppose that $F(A)(\omega) \neq 0$ and $\sum_{i \in \mathbf{Z}}\left|a_{i, 0}\right|<\infty$. It is known from the well-known Wiener's theorem that there exists a sequence $\mathbf{x}$ such that

$$
\frac{1}{F(A)(\omega)}=\sum_{k \in \mathbf{Z}} x_{k} e^{-j k \omega}
$$

with $\sum_{k}\left|x_{k}\right|<\infty$. It is easy to see that to find this sequence $\mathbf{x}$ is equivalent to solving the linear system of bi-infinite order:

$$
A \mathbf{x}=\delta,
$$


where $\delta=\left(\ldots, \delta_{-1}, \delta_{0}, \delta_{1}, \ldots\right)^{t}$ with $\delta_{0}=1$ and $\delta_{i}=0$ for all $i \in \mathbf{Z} \backslash\{0\}$.

Furthermore, if the matrix $A$ is a positive operator, then there exists a unique positive square root $P$ of $A$. That is, $P^{2}=A$. The symbol representation is $F(P)(\omega)=\sqrt{F(A)(\omega)}$. To find $F(P)(\omega)$ is equivalent to finding a matrix $P$ such that $P^{2}=A$.

Certainly, we cannot solve a linear system of bi-infinite order. Neither can we decompose a matrix of bi-infinite order into two matrices of bi-infinite order. However, we can do this approximatively. Let $N$ be a positive integer, and let $A_{N}=\left(a_{i k}\right)_{-N<i, k \leq N}$ be a finite section of $A$. Let $I_{N, \infty}=\left(0, I_{2 N+1,2 N+1}, 0\right)$ be a matrix of $2 N+1$ rows and bi-infinite columns with $I_{2 N+1,2 N+1}$ being the identity matrix of size $(2 N+1) \times(2 N+1)$ such that

$$
A_{N}=I_{N, \infty} A I_{N, \infty}^{t} \text {. }
$$

Denote $\delta_{N}=I_{N, \infty} \delta$ and $\mathbf{x}_{N}=I_{N, \infty} \mathbf{x}$. Then we shall solve the following linear system:

$$
A_{N} \hat{\mathbf{x}}_{N}=\delta_{N} .
$$

We claim that $\hat{\mathbf{x}}_{N}$ converges to $\mathbf{x}$ exponentially fast as $N$ increases to $\infty$, under certain conditions on $A$. Furthermore, we shall solve $\widehat{P}_{N}^{2}=A_{N}$ for $\widehat{P}_{N}$ by using the singular value decomposition. Once we have $\widehat{P}_{N}$, we shall solve

$$
\widehat{P}_{N} \hat{\mathbf{y}}_{N}=\delta_{N} .
$$

We claim that $\hat{\mathbf{y}}_{N}$ converges to $\mathbf{y}$ exponentially fast as $N \rightarrow \infty$, provided $A$ satisfies certain conditions.

To check the conditions on $A$, we need the following definition.

Definition. A matrix $A=\left(a_{i k}\right)_{i, k \in \mathbf{Z}}$ is said to be of exponential decay off its diagonal if

$$
\left|a_{i k}\right| \leq K r^{|i-k|}
$$

for some constant $K$ and $r \in(0,1)$.

We begin with an elementary lemma.

Lemma 1. Suppose that $A$ is of exponential decay off its diagonal and has a bounded inverse. Suppose that $A_{N}^{-1}=\left(\hat{a}_{i k}\right)_{-N \leq i, k \leq N}$ satisfies the property that

$$
\left|\hat{a}_{i, k}(N)\right| \leq K r^{|i-k|}, \quad \forall-N \leq i, k \leq N,
$$

for all $N>0$. Then there exists $r_{1} \in(0,1)$ and a constant $K_{1}$ such that

$$
\left\|I_{N, \infty} \mathbf{x}-\hat{\mathbf{x}}_{N}\right\|_{2} \leq K_{1} r_{1}^{N},
$$

where $\mathbf{x}$ is the solution of $A \mathbf{x}=\delta$ and $\mathbf{x}_{N}$ is the solution of $A_{N} \mathbf{x}_{N}=\delta_{N}$.

Proof. From the assumption of the lemma, there exist $K$ and $r \in(0,1)$ such that $A=\left(a_{i k}\right)_{i, k \in \mathbf{Z}}$ and $A_{N}^{-1}=\left(\hat{a}_{i, k}(N)\right)_{-N \leq i, k \leq N}$ satisfy

$$
\left|a_{i k}\right| \leq K r^{|i-k|} \text { and }\left|\hat{a}_{i k}\right| \leq K r^{|i-k|} \text {. }
$$

Write

$$
A I_{N, \infty}^{t}=\left[\begin{array}{c}
B \\
A_{N} \\
C
\end{array}\right] \quad \text { and } \quad d=B A_{N}^{-1} \delta_{N} \quad \text { with } d=\left(\ldots, d_{-N-1}, d_{-N}\right)^{t}
$$


Then we have, for each $i=-\infty, \ldots,-N-1,-N$,

$$
\begin{aligned}
\left|d_{i}\right| & =\left|\sum_{k=-N}^{N} a_{i k} \hat{a}_{k, 0}(N)\right| \leq K^{2} \sum_{k=-N}^{N} r^{|i-k|} r^{|k|} \\
& =K^{2}\left(r^{-i} \sum_{k=0}^{N} r^{2 k}+N r^{-i}\right) \leq C \lambda^{-i}
\end{aligned}
$$

for some constant $C$ and $\lambda \in(0,1)$. Thus, $\left\|B A^{-1} \delta_{N}\right\|_{2} \leq C^{\prime} \lambda^{N}$. Similarly, $\left\|C A_{N}^{-1} \delta_{N}\right\|_{2} \leq C^{\prime} \lambda^{N}$. Hence,

$$
\begin{aligned}
\left\|I_{N, \infty} \mathbf{x}-\hat{\mathbf{x}}_{N}\right\|_{2} & \leq\left\|\mathbf{x}-I_{N, \infty}^{t} \hat{\mathbf{x}}_{N}\right\|_{2} \leq\left\|A^{-1}\right\|_{2}\left\|\delta-A I_{N, \infty}^{t} A_{N}^{-1} \delta_{N}\right\|_{2} \\
& \leq\left\|A^{-1}\right\|_{2}\left\|\delta-\left[\begin{array}{c}
B \\
A_{N} \\
C
\end{array}\right] A_{N}^{-1} \delta_{N}\right\|_{2} \\
& \leq\left\|A^{-1}\right\|_{2}\left\|\delta-\left[\begin{array}{c}
B A_{N}^{-1} \\
I_{2 N+1,2 N+1} \\
C A_{N}^{-1}
\end{array}\right] \delta_{N}\right\|_{2} \\
& \leq\left\|A^{-1}\right\|_{2}\left(\left\|B A_{N}^{-1} \delta_{N}\right\|_{2}+\left\|C A_{N}^{-1} \delta_{N}\right\|_{2}\right) \leq\left\|A^{-1}\right\|_{2} 2 C^{\prime} \lambda^{N},
\end{aligned}
$$

hence the assertion with $K_{1}=2 C^{\prime}\left\|A^{-1}\right\|_{2}$ and $r_{1}=\lambda$. This establishes the lemma.

Next, we consider approximating the square root of a positive operator.

Lemma 2. Let $P$ be the unique square root of a positive operator $A$. Suppose that $A$ is banded and $\|A-I\|_{2} \leq r<1$, where $I$ is the identity operator from $l^{2}$ to $l^{2}$. Then $P=\left(p_{i k}\right)_{i, k \in \mathrm{Z}}$ is of exponential decay off its diagonal.

Proof. The uniqueness of $P$ and the convergence of the series

$$
\sum_{i=0}^{\infty}(-1)^{i} \frac{(2 i-3) ! !}{(2 i) ! !}(A-I)^{i}
$$

imply that

$$
P=\sqrt{A}=\sqrt{I+(A-I)}=\sum_{i=0}^{\infty}(-1)^{i} \frac{(2 i-3) ! !}{(2 i) ! !}(A-I)^{i} .
$$

The matrix $A$ is banded and so is $A-I$. If $A-I$ has bandwidth $b$, then $(A-I)^{i}$ is also banded with bandwidth $i b$. Thus, $\left|p_{i k}\right| \leq K r^{|i-k| / b}$ for some constant $K$. This finishes the proof.

Lemma 3. Let $P$ be the unique square root of a positive operator $A$. Suppose that $A$ is banded and $\|A-I\|_{2} \leq r<1$, where $I$ is the identity operator from $l^{2}$ to $l^{2}$. Then $P^{-1}=\left(\hat{p}_{i k}\right)_{i, k \in \mathrm{Z}}$ is of exponential decay off its diagonal.

Proof. The uniqueness of $P^{-1}$ and the convergence of the series

$$
\sum_{i=0}^{\infty}(-1)^{i} \frac{(2 i-1) ! !}{(2 i) ! !}(A-I)^{i}
$$


imply that

$$
P^{-1}=(A)^{-1 / 2}=(I+(A-I))^{-1 / 2}=\sum_{i=0}^{\infty}(-1)^{i} \frac{(2 i-1) ! !}{(2 i) ! !}(A-I)^{i} .
$$

Now we use the same argument as in the lemma above to conclude that $P^{-1}$ is of exponential decay off its diagonal.

Let $\widehat{P}_{N}$ be the square root of $A_{N}$. That is, $\widehat{P}_{N}^{2}=A_{N}$. Denote $P_{N}=$ $I_{N, \infty} P I_{N, \infty}^{t}$. We need to estimate $P_{N} \widehat{P}_{N}-\widehat{P}_{N} P_{N}$. We have

Lemma 4. Let $R=\left(r_{i k}\right)_{-N \leq i, k \leq N}:=P_{N} \widehat{P}_{N}-\widehat{P}_{N} P_{N}$. Then $r_{i k}=O\left(r^{N /(4 b)}\right)$ for $k=-N / 4+1, \ldots, N / 4-1$ and $i=-N, \ldots, N$, where $b$ is the bandwidth of $A$ and $r$ is as defined in Lemma 3.

Proof. It is known that $P$ and $A$ commute. Let us write

$$
P=\left[\begin{array}{ccc}
\alpha_{1} & B & \alpha_{2} \\
B^{t} & P_{N} & C^{t} \\
\alpha_{3} & C & \alpha_{4}
\end{array}\right] \text { and } A=\left[\begin{array}{ccc}
\beta_{1} & a & \beta_{2} \\
a^{t} & A_{N} & c^{t} \\
\beta_{3} & c & \beta_{4}
\end{array}\right] .
$$

We have $B^{t} a+P_{N} A_{N}+C^{t} c=a^{t} B+A_{N} P_{N}+c^{t} C$. Thus, $P_{N} A_{N}-A_{N} P_{N}=$ $a^{t} B-B^{t} a+c^{t} C-C^{t} c$. Let $E=a^{t} B-B^{t} a+c^{t} C-C^{t} c$ and $I_{N}:=I_{2 N+1,2 N+1}$. We have $P_{N}\left(A_{N}-I_{N}\right)=\left(A_{N}-I_{N}\right) P_{N}+E$ and

$$
P_{N}\left(A_{N}-I_{N}\right)^{n}=\left(A_{N}-I_{N}\right)^{n} P_{N}+\sum_{k=0}^{n-1}\left(A_{N}-I_{N}\right)^{k} E\left(A_{N}-I_{N}\right)^{n-k-1}
$$

by using induction. Then, we have

$$
\begin{aligned}
P_{N} \widehat{P}_{N}= & \sum_{n=0}^{\infty}(-1)^{n} \frac{(2 n-3) ! !}{(2 n) ! !} P_{N}\left(A_{N}-I_{N}\right)^{n} \\
= & \sum_{n=0}^{\infty}(-1)^{n} \frac{(2 n-3) ! !}{(2 n) ! !}\left(A_{N}-I_{N}\right)^{n} P_{N} \\
& +\sum_{n=0}^{\infty}(-1)^{n} \frac{(2 n-3) ! !}{(2 n) ! !} \sum_{k=0}^{n-1}\left(A_{N}-I_{N}\right)^{k} E\left(A_{N}-I_{N}\right)^{n-k-1} \\
= & \widehat{P}_{N} P_{N}+\sum_{n=0}^{\infty}(-1)^{n} \frac{(2 n-3) ! !}{(2 n) ! !} \sum_{k=0}^{n-1}\left(A_{N}-I_{N}\right)^{k} E\left(A_{N}-I_{N}\right)^{n-k-1} .
\end{aligned}
$$

To estimate $R=P_{N} \widehat{P}_{N}-\widehat{P}_{N} P_{N}$ which is the summation above, we break $R$ into two parts and estimate the first by

$$
\begin{aligned}
& \left\|\sum_{n=N 1+1}^{\infty}(-1)^{n} \frac{(2 n-3) ! !}{(2 n) ! !} \sum_{k=0}^{n-1}\left(A_{N}-I_{N}\right)^{k} E\left(A_{N}-I_{N}\right)^{n-k-1}\right\|_{2} \\
& \quad \leq \sum_{n=N 1+1}^{\infty} \frac{(2 n-3) ! ! n}{(2 n) ! !}\|E\|_{2}\left\|A_{N}-I_{N}\right\|_{2}^{n} \leq K_{1}\left\|A_{N}-I_{N}\right\|_{2}^{N_{1}} .
\end{aligned}
$$

Thus, this part has the desired property if we choose $N 1$ appropriately. Next, we note that $A_{N}-I_{N}$ is banded and its bandwidth is $b$. Thus, for $0 \leq n \leq N 1$, $\left(A_{N}-I_{N}\right)^{n}$ is also banded and has bandwidth $n b \leq b N 1$. 
Note also $E=\left(e_{i k}\right)_{-N \leq i, k \leq N}$ has the following property:

$e_{i k}= \begin{cases}0 & \text { for }-N+b<k<N-b,-N+b<i<N-b, \\ O\left(r^{N-|k|}\right) & \text { for }-N \leq i \leq-N+b \text { and } N-b \leq i \leq N,-N \leq k \leq N .\end{cases}$

It follows that $\left(A_{N}-I_{N}\right)^{l} E$ has a similar property as $E$ :

$$
\left(\left(A_{N}-I_{N}\right)^{l} E\right)_{i k}= \begin{cases}0 & \text { for }-N+b<k<N-b, \\ & -N+k b+b<i<N-l b-b, \\ O\left(r^{N-|k|}\right) & \text { for }-N \leq i \leq-N+l b+b, \\ & N-l b-b \leq i \leq N, \text { and }-N \leq k \leq N .\end{cases}
$$

Choose $N 1$ such that $N /(4 b) \leq N 1<N /(4 b)+1$. Then $\left(A_{N}-I\right)^{N 1}$ has bandwidth $b N 1<N / 4+b$ and hence

$\left(\left(A_{N}-I\right)^{l} E\left(A_{N}-I\right)^{n-l-1}\right)_{i k}= \begin{cases}O\left(r^{3 N / 4-b-|k|}\right) & \text { if }|k| \leq N / 4 \text { and }-N \leq i \leq N, \\ O(1) & \text { otherwise }\end{cases}$

for $l=1, \ldots, N 1$. Putting these two parts together, we have established that $R$ has the desired property.

We are now ready to prove the following.

Theorem 1. Suppose that $A$ is a positive operator and $\|A-I\|_{2}<1$. Suppose that $A$ is a banded matrix. Let $P$.be the unique square root of $A$ and $\mathbf{y}$ the solution of $P \mathbf{y}=\delta$. Let $\widehat{P}_{N}$ be a square root matrix such that $\widehat{P}_{N}^{2}=A_{N}$ and $\hat{\mathbf{y}}_{N}$ the solution of $\widehat{P}_{N} \hat{\mathbf{y}}_{N}=\delta_{N}$. Then

$$
\left\|I_{N, \infty} \mathbf{y}-\hat{\mathbf{y}}_{N}\right\|_{2} \leq K \lambda^{N}
$$

for some $\lambda \in(0,1)$ and a constant $K>0$.

Proof. Let $P=\left(p_{i k}\right)_{i, k \in Z}$ and $P_{N}=\left(p_{i k}\right)_{-N \leq i, k \leq N}$. By Lemma 2, the matrix $P$ is of exponential decay off its diagonal. By Lemma 3, we know that $P_{N}$ is of exponential decay off its diagonal uniformly with respect to $N$ because of $\left\|A_{N}-I_{2 N+1,2 N+1}\right\|_{2}<1$, which follows from $\|A-I\|_{2}<1$. The invertibility of $A$ implies that $P$ is invertible. From $\|A-I\|_{2}<1$ it follows that the inverse of $P$ is bounded. Let $\tilde{\mathbf{y}}_{N}$ be the solution of $P_{N} \tilde{\mathbf{y}}_{N}=\delta_{N}$. Thus, we apply Lemma 1 to conclude that

$$
\left\|I_{N, \infty} \mathbf{y}-\tilde{\mathbf{y}}_{N}\right\|_{2} \leq K_{1} r^{N}
$$

for some $r \in(0,1)$.

We now proceed to estimate $\left\|\tilde{\mathbf{y}}_{N}-\hat{\mathbf{y}}_{N}\right\|_{2}$.

Note that $P^{2}=A$ implies $A_{N}=P_{N}^{2}+B^{t} B+C^{t} C$ or $\widehat{P}_{N}^{2}-P_{N}^{2}=B^{t} B+C^{t} C$. Thus, we have

$$
\left(P_{N}+\widehat{P}_{N}\right)\left(\widehat{P}_{N}-P_{N}\right)=\widehat{P}_{N}^{2}-P_{N}^{2}+P_{N} \widehat{P}_{N}-\widehat{P}_{N} P_{N}=B^{t} B+C^{t} C+R,
$$

where $R$ was defined in Lemma 4 . Hence,

$$
\left(\widehat{P}_{N}-P_{N}\right)=\left(P_{N}+\widehat{P}_{N}\right)^{-1}\left(B^{t} B+C^{t} C+R\right) \text {. }
$$

Note that the entries of $B^{t} B+C^{t} C$ have the exponential decay property: $\left(B^{t} B+C^{t} C\right)_{i k}=O\left(r^{N-|k|}\right)$. By Lemma 4, we know that each entry of the middle section $\left(N / 2\right.$ columns) of the columns of $B^{t} B+C^{t} C+R$ has exponential 
decay $O\left(r^{N /(4 b)}\right)$. Both $P_{N}$ and $\widehat{P}_{N}$ are positive and $\left\|\left(P_{N}+\widehat{P}_{N}\right)^{-1}\right\|_{2} \leq\left\|\widehat{P}_{N}^{-1}\right\|_{2}$ is bounded. Recall that $P_{N}^{-1}$ is of exponential decay off its diagonal. We have

$$
\begin{aligned}
\left\|\tilde{\mathbf{y}}_{N}-\hat{\mathbf{y}}_{N}\right\|_{2} & \leq\left\|\widehat{P}_{N}^{-1}\right\|_{2}\left\|\delta_{N}-\widehat{P}_{N} P_{N}^{-1} \delta_{N}\right\|_{2} \\
& \leq\left\|\widehat{P}_{N}^{-1}\right\|_{2}\left\|\left(P_{N}-\widehat{P}_{N}\right)\left(P_{N}^{-1} \delta_{N}\right)\right\|_{2} \\
& \leq\left\|\widehat{P}_{N}^{-1}\right\|_{2}\left\|\left(P_{N}+\widehat{P}_{N}\right)^{-1}\right\|_{2}\left\|\left(B^{t} B+C^{t} C+R\right) P_{N}^{-1} \delta_{N}\right\|_{2} \\
& \leq K \lambda^{N}
\end{aligned}
$$

for some $\lambda \in(r, 1)$. This completes the proof.

In the proof above, an essential step is to show that each entry of the middle section of the columns of $\widehat{P}_{N}-P_{N}$ is of exponential decay. This indeed follows from $\left(\widehat{P}_{N}-P_{N}\right)=\left(P_{N}+\widehat{P}_{N}\right)^{-1}\left(B^{t} B+C^{t} C+R\right)$, the boundedness of $\left(P_{N}+\widehat{P}_{N}\right)^{-1}$, and the fact that each entry of the middle section of the columns of $B^{t} B+C^{t} C+R$ is of exponential decay. This has its own interest. Thus, we have the following

Theorem 2. Suppose that $A$ is a positive operator and $\|A-I\|_{2}<1$. Suppose that $A$ is a banded matrix. Let $P$ be the unique square root of $A$ and $P_{N}=$ $I_{N, \infty} P\left(I_{N, \infty}\right)^{t}$. Let $\widehat{P}_{N}$ be a square root matrix such that $\widehat{P}_{N}^{2}=A_{N}$. Then

$$
\left\|P_{N} \delta_{N}-\widehat{P}_{N} \delta_{N}\right\|_{2} \leq K \lambda^{N}
$$

for some $\lambda \in(0,1)$ and a constant $K$.

Finally, we remark that if $\|A-I\|_{2}=1$, then each entry of the middle section of the columns of $R$ is convergent to 0 with speed $\frac{1}{N}$. The exponential decay in the above has to be replaced by

$$
\left\|P_{N} \delta_{N}-\widehat{P}_{N} \delta_{N}\right\|_{2} \leq \frac{K}{N} .
$$

\section{Computation of Battle-Lemariés wavelets}

Fix a positive integer $m$. Let $A=\mathbf{B}_{2 m}$ be the bi-infinite matrix whose symbol is $\sum_{k \in \mathbf{Z}} B_{2 m}(k) e^{-j k \omega}$. Clearly, $A$ is a banded Toeplitz matrix. To see that $A$ is a positive operator on $l^{2}$, we show that $A \geq c I$ for some $c>0$ as follows: For any $\mathbf{x} \in l^{2}$, we have

$$
\begin{aligned}
\mathbf{x}^{t} A \mathbf{x} & =\frac{1}{2 \pi} \int_{-\pi}^{\pi} \overline{F(\mathbf{x})(\omega)} F(A)(\omega) F(\mathbf{x})(\omega) d \omega \\
& =F(A)(\xi) \frac{1}{2 \pi} \int_{-\pi}^{\pi}|F(\mathbf{x})(\omega)|^{2} d \omega \\
& \geq \min _{\omega} F(A)(\omega)\|\mathbf{x}\|_{2}^{2} .
\end{aligned}
$$

With $c=\min _{\omega} F(A)(\omega)>0$, we have $A \geq c I$. Similarly, we can show that 
$\|A-I\|_{2}<1$. Indeed,

$$
\begin{aligned}
\|(A-I) \mathbf{x}\|_{2}^{2}=\frac{1}{2 \pi} \int_{-\pi}^{\pi}|F(A-I)(\omega)|^{2}|F(\mathbf{x})(\omega)|^{2} d \omega \\
\quad=\frac{1}{2 \pi} \int_{-\pi}^{\pi}|1-F(A)(\omega)|^{2}|F(\mathbf{x})(\omega)|^{2} d \omega \\
\quad \leq \max _{\omega}|1-F(A)(\omega)|^{2}\|\mathbf{x}\|_{2}^{2} \leq\left(1-\min _{\omega} F(A)(\omega)\right)^{2}\|\mathbf{x}\|_{2}^{2} .
\end{aligned}
$$

Thus, we have

$$
\|(A-I) \mathbf{x}\|_{2} \leq\left(1-\min _{\omega} F(A)(\omega)\right)\|\mathbf{x}\|_{2}
$$

and hence, $\|A-I\|_{2}<1$. Thus, $\mathbf{B}_{2 m}$ satisfies all the conditions of Theorem 1 .

By (1), we have

$$
\hat{\varphi}(\omega)=\frac{1}{\sqrt{\sum_{k \in \mathbf{Z}} B_{2 m}(k) e^{-j k \omega}}}\left(\frac{\sin \omega / 2}{\omega / 2}\right)^{m} .
$$

Thus, $\varphi(t)=\sum_{k} \alpha_{k} B_{m}(t-k)$ with $\mathbf{x}=\left(\alpha_{k}\right)_{k \in \mathbf{Z}}$ satisfying

$$
\mathbf{C}_{2 m} \mathbf{x}=\delta \quad \text { and } \quad \mathbf{C}_{2 m}^{2}=\mathbf{B}_{2 m} .
$$

Using our Theorem 1, we conclude that our numerical method is valid to compute the $\alpha_{k}$ 's.

By (2), the transfer function is

$$
H(\omega)=\frac{\sqrt{\sum_{k \in \mathbf{Z}} B_{2 m}(k) e^{-j 2 k \omega}}}{\sqrt{\sum_{k \in \mathbf{Z}} B_{2 m}(k) e^{-j k \omega}}} \cos ^{m}(\omega / 2) .
$$

Note that when $m$ is even, then $\cos ^{m}(\omega / 2)=\left(1-\left(e^{j \omega}+e^{-j \omega}\right) / 2\right)^{m / 2}$, which is a finite series. However, when $m$ is odd, $\cos ^{m}(\omega / 2)$ is no longer a finite series. In order to compute $H(\omega)$, let $\mathbf{S}_{m}$ be the Toeplitz matrix whose symbol is $\cos ^{2 m}(\omega / 2)=\left(1-\left(e^{j \omega}+e^{-j \omega}\right) / 2\right)^{m}$. Let $Z$ be a zero insertion operator on $l^{2}$ defined by

$$
Z \mathbf{x}=Z\left(x_{i}\right)_{i \in \mathbf{Z}}=\left(z_{i}\right)_{i \in \mathbf{Z}} \quad \text { with } z_{i}= \begin{cases}x_{i / 2} & \text { if } i \text { is even, } \\ 0 & \text { if } i \text { is odd } .\end{cases}
$$

Thus, $H(\omega)=\sum_{k \in \mathbf{Z}} \beta_{k} e^{-j k \omega}$ with $\mathbf{x}=\left(\beta_{k}\right)_{k \in \mathbf{Z}}$ satisfying

$$
\mathbf{x}=\mathbf{w} * \mathbf{y} * \mathbf{z},
$$

where $*$ denotes the convolution operator of two vectors in $l^{2}$ and

$$
\mathbf{y}=\mathbf{C}_{m} \delta, \quad \mathbf{z}=Z \mathbf{C}_{m}^{-1} \delta, \quad \mathbf{w}=\mathbf{T} \delta
$$

with $\mathbf{C}_{m}^{2}=\mathbf{B}_{2 m}, \mathbf{T}_{m}^{2}=\mathbf{S}_{m}$. Using our Theorems 1 and 2, we know that our numerical method gives a good approximation to $\mathbf{y}$ and $\mathbf{z}$. For $m$ even, our numerical method produces an $\mathbf{x}_{N}$ which converges pointwise to $\mathbf{x}$ exponentially. When $m$ is odd, the remark after Theorem 2 has to be applied, and the $\mathbf{w}_{N}$ produced by this procedure does no longer converge to $\mathbf{w}$ exponentially. 
By (3), the wavelet $\psi$ associated with $\varphi$ is given by

$$
\hat{\psi}(2 \omega)=e^{-j \omega} \overline{H(\omega+\pi)} \hat{\varphi}(\omega) .
$$

Once $\left\{\alpha_{k}\right\}_{k \in \mathbf{Z}}$ and $\left\{\boldsymbol{\beta}_{k}\right\}_{k \in \mathbf{Z}}$ are computed, $\left\{\gamma_{k}\right\}_{k \in \mathbf{Z}}$ can be obtained by convolution.

We have implemented this method to compute Battle-Lemariés wavelets in MATLAB. The graphs of Battle-Lemarié's wavelets are shown in the following figures.
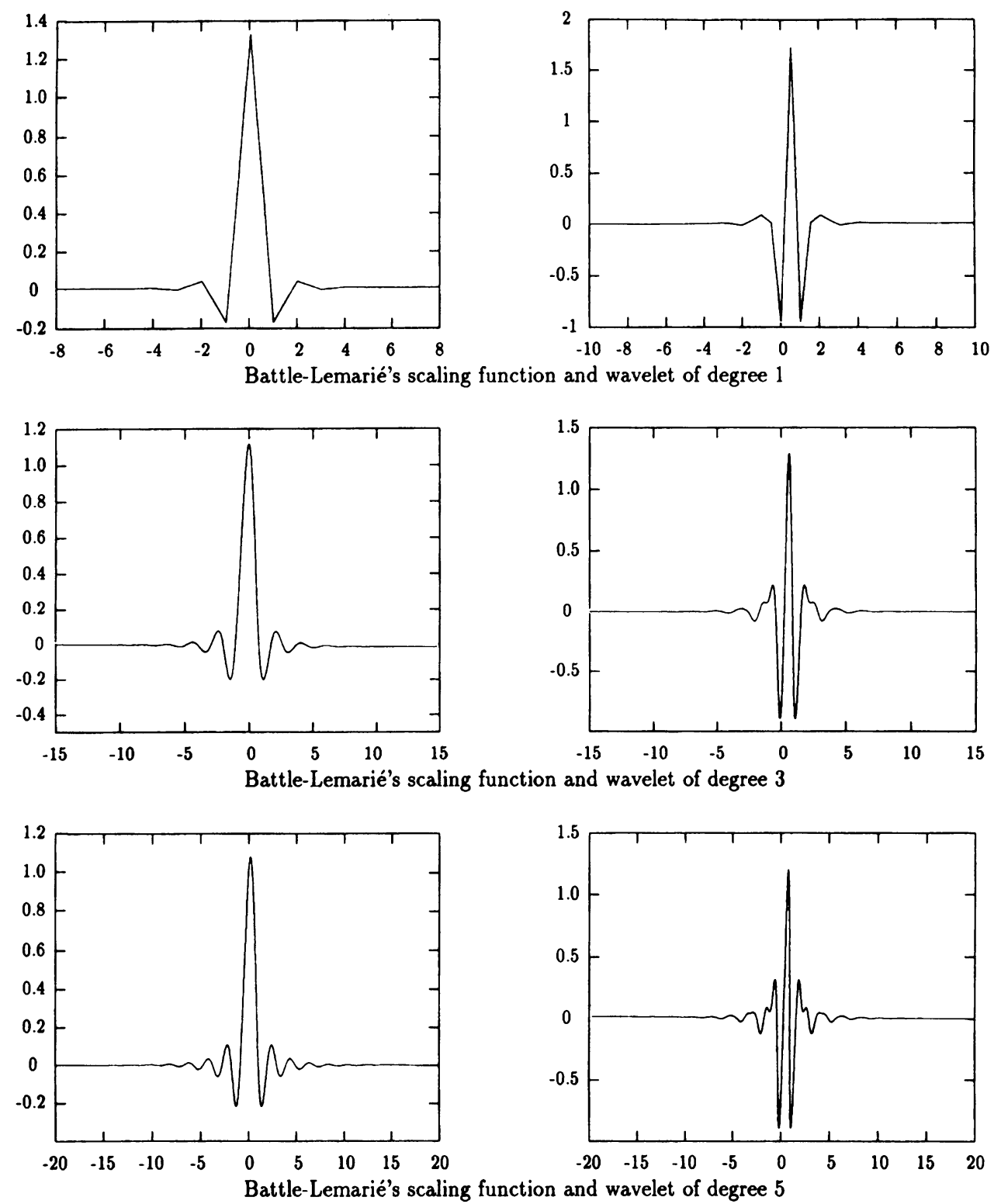

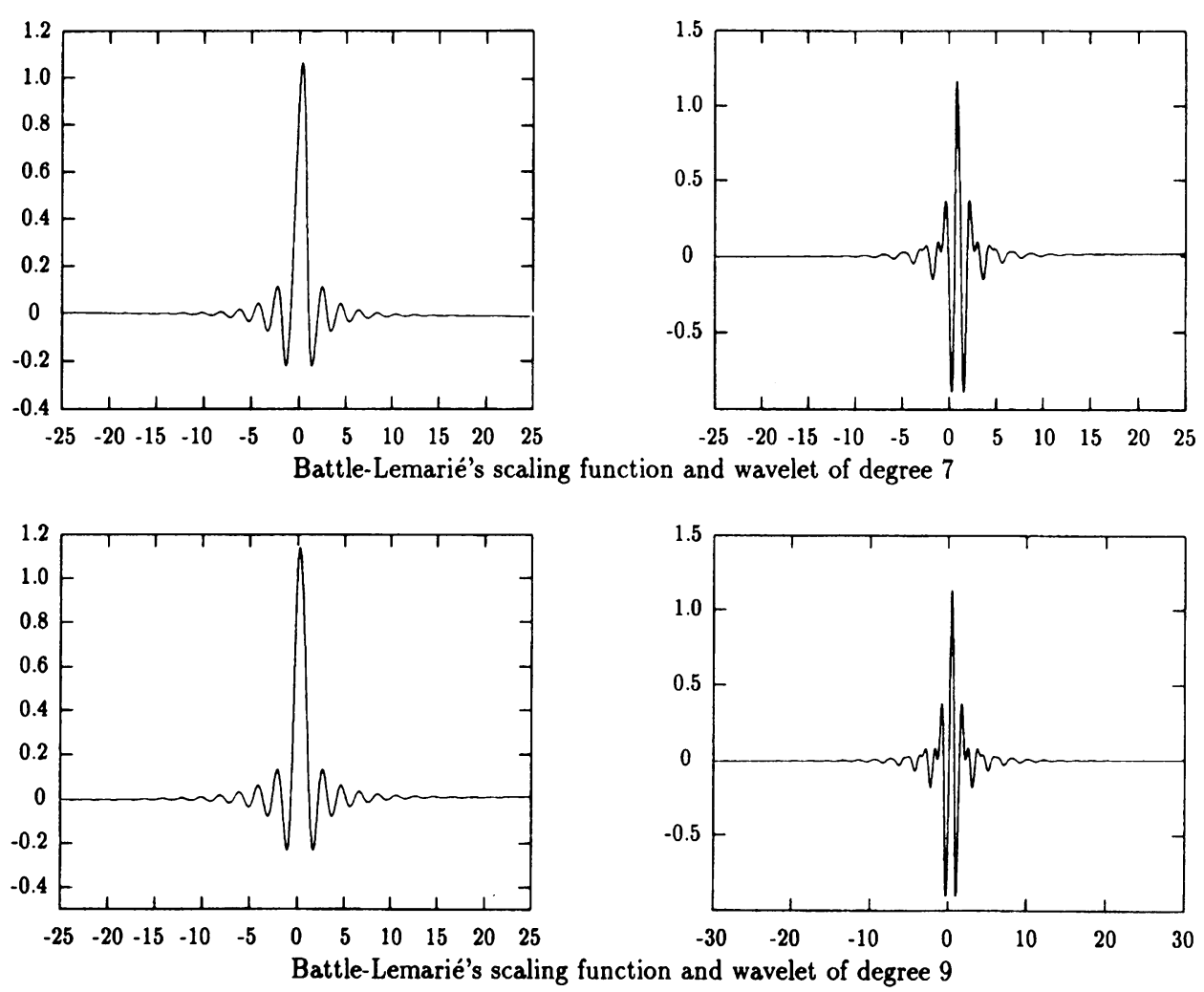

\section{BIBLIOGRAPHY}

1. G. Battle, A block spin construction of ondelettes, I: Lemarié functions, Comm. Math. Phys. 110 (1987), 601-615.

2. I. Daubechies, Ten lectures on wavelets, SIAM, Philadelphia, PA, 1992.

3. P.-G. Lemarié, Ondelettes à localisation exponentielles, J. Math. Pures Appl. (9) 67 (1988), 227-236.

4. S. Mallat, Multiresolution approximations and wavelet orthonormal bases of $L^{2}(\mathbf{R})$, Trans. Amer. Math. Soc. 315 (1989), 69-87.

5. __ A theory for multiresolution signal decomposition: the wavelet representation, IEEE Trans. Pattern Anal. and Machine Intelligence 11 (1989), 674-693.

Department of Mathematics, University of Georgia, Athens, Georgia 30602

E-mail address: mjlai@wiener.math.uga.edu 